\title{
Linearization Method of Carrier Catalytic Gas Sensor Characteristics Us- ing High-Order Polynomial
}

\author{
Huang Weiyong ${ }^{1, *}$, Gao Yuqing ${ }^{2}$ and Tian Xiuling ${ }^{3}$ \\ ${ }^{I}$ Xuzhou Key Laboratory of Virtual Reality and Multi-dimensional Information Processing, Xuzhou Institute of Technol- \\ ogy, Xuzhou Jiangsu, P.R. China \\ ${ }^{2}$ Jiangsu Key Laboratory of Large Engineering Equipment Detection and Control, Xuzhou Institute of Technology, \\ Xuzhou Jiangsu, P.R. China \\ ${ }^{3}$ School of Information and Electrical Engineering, Xuzhou Institute of Technology, Xuzhou Jiangsu, P.R. China
}

\begin{abstract}
To improve detecting performance of gas concentration in coal-mine, a new linearization method of carrier catalytic gas sensor characteristics based on high-order polynomials was introduced. Firstly, the calibration data of sensor was used to establish the training sample set. Then, the high-order polynomial was employed to establish nonlinear regression inverse model of sensor characteristics. Finally, the polynomial coefficients were intelligently tuned by improved clone selection algorithm (ICSA) and the criteria of mean absolute error (MAE) minimization. Experimental results showed that the method proposed in this paper is effective, and the performance of linearization model is superior to that of the traditional least square method.
\end{abstract}

Keywords: Carrier catalytic gas sensor, Linearization, High-order polynomial, Clone selection algorithm.

\section{INTRODUCTION}

Nowadays carrier catalytic gas sensor plays an important role in coal-mine gas measurement systems. Due to the nonlinear characteristics of carrier catalytic gas sensors, external environment, electronic component aging, and many other uncertain factors, there is a complex nonlinear relationship between the actual output voltage of sensor and gas concentration [1]. Because the nonlinear characteristics of carrier catalytic sensor directly affect and even determine the performance of the gas measurement system, seeking an effective mathematical model to realize the linearization of carrier catalytic gas sensor characteristics is of great significance, so as to ensure the measurement accuracy of gas concentration in coal-mine.

Considering that traditional methods, such as look-up table, piecewise linearization and so on, have the shortcoming of low accuracy and they can not be applicable to high accuracy occasions, many researchers have carried on the thorough research in the field of sensor characteristics linearization. CHEN proposed a least-squares method [2], but the method was based on the descent of sum of error square to find the optimal solution, easily trapping in local minimum point and not getting the global optimal solution. Especially when the data points are larger, the method is prone to oscillation phenomenon, even unable to obtain polynomial

*Address correspondence to this author at the School of Information and Electrical Engineering, Xuzhou Institute of Technology, Jiangsu, 221111, P.R. China; Tel: 13776786967; E-mail: h_weiyongs@163.com coefficient [3]. Liu proposed a kind of linearization method based on neural network [4]. Because neural network is based on the law of large numbers using the empirical risk criterion with the complex model to fit the limited samples, the linearization model using neural network is unstable and has poor generalization ability [5]. In order to get higher precision, Sun proposed a kind of least squares support vector machines (LSSVM) method [6], but this method is difficult to be directly applied in the detecting systems of mine gas. Both theory and practice show that the high-order polynomial can fit any nonlinear curve, and is easy to be implemented in the detecting devices with single-chip microcomputer.

The current paper adopts high-order polynomial for linearization of catalytic gas sensor characteristics, so as to get better detecting performance. As the polynomial coefficients determine directly the polynomial performance, the selection and optimization of polynomial coefficient has become an important issue to be resolved. Clone selection algorithm (CSA) [7] by Castro according to the theory of immunity in the clone selection mechanism is a new optimization algorithm with heuristic intelligence, which not only has a faster convergence speed in the search range, but also can overcome the problem of premature convergence that many other evolutionary computation algorithms have [8]. Clone selection algorithm has achieved excellent results in many practical applications with the superior performances to the classical approaches, and also provides a new way for the selection and optimization of high-order polynomial coefficient. 
The output error of linearization model is an important evaluation criteria reflecting the system accuracy, and the mean square error (MSE) is usually used in the optimization of polynomial coefficient optimization. Research shows that when optimizing high-order polynomial coefficient, adopting MSE evaluation criteria has some deficiencies: when outliers or singular points exist in the sample set, their effects will be magnified, which can reduce overall modeling accuracy of the system. While the evaluation criteria of mean absolute error (MAE) minimization is applied, the robustness of linearization model system is better [9].

To this end, a new linearization method of carrier catalytic gas sensor characteristics by using high-order polynomial and an improved clone selection algorithm (ICSA) was introduced in an attempt to tune intelligently the polynomial coefficients. The experimental results showed that the proposed method in this paper is feasible, and can yield better performance than the conventional least square method.

This paper is organized as follows: Section 1 outlines briefly the related work. Section 2 states the linearization principle of sensor characteristics using high-order polynomial. Section 3 presents the principle and algorithm of ICSA. The procedure for selecting and optimizing polynomial coefficient based on ICSA is presented in Section 4, followed by the experiment results and the conclusions in Section 5 and in Section 6.

\section{LINEARIZATION MODEL OF SENSOR USING HIGH-ORDER POLYNOMIAL}

The linearization of catalytic gas sensor characteristics using high-order polynomial is to establish an inverse model of the sensor characteristics using multiple sensor calibration data by adjusting the polynomial coefficients to find the minimum difference between the model output and the test data. If the hysteresis and creep effect of sensor are not considered, the linearization model of catalytic gas sensor characteristics can be described with n-order polynomial as follows:

$$
\hat{\mathrm{x}}=\mathrm{a}_{\mathrm{n}} \mathrm{u}^{\mathrm{n}}+\mathrm{a}_{\mathrm{n}-1} \mathrm{u}^{\mathrm{n}-1}+\cdots+\mathrm{a}_{2} \mathrm{u}^{2}+\mathrm{a}_{1} \mathrm{u}+\mathrm{a}_{0}
$$

where, $\mathrm{u}$ is the output voltage of sensor, $\hat{x}$ is measurement

value of gas concentration, $\mathrm{n}$ is the order of polynomial, $\mathrm{a}_{\mathrm{i}}$ $(i=n, n-1, \ldots 2,1,0)$ is polynomial coefficient to be selected and optimized.

Due to $a_{i}$ in formula (1) reflecting the inverse characteristics of sensor, how to determine the $a_{i}$ values become the key to implement the characteristics linearization of catalytic gas sensor.

By calibrating the sensor to obtain input/output data of sensor, researchers used to select the polynomial coefficients using least square method. The polynomial coefficients were stored in single-chip microcomputer and when the output voltage of sensor was detected, the real value of gas could be calculated by Formula (1). Because the principle of least square method is simple and its programming is not difficult, the least square method has become an important means for parameters estimation by experimental data.
As mentioned above, the least square method in many cases can not obtain the optimal solution, and even is unable to obtain the polynomial coefficients that we want to obtain. The development of intelligent optimization technology makes it possible for people to tune parameters of mathematical model, which can make the established model more credible [10]. Clone selection algorithm was adopted in this paper to select and optimize the polynomial coefficient, so as to get better detecting results.

\section{IMPROVED CLONE SELECTION ALGORITHM}

The basic principle of clone selection algorithm is that the function to be optimized and its constraints is viewed as antigen, the solution of the problem is viewed as antibody and the affinity between the antibody, antibody-antigen is viewed as the objective function of the problem [11]. In order to tune automatically the polynomial coefficients, an improved clone selection algorithm is outlined in the paper. Its block diagram is shown in Fig. (1). The corresponding steps are explained in detail as follows:

Step 1: Initialization: Randomly generate $\mathrm{n}$ antibodies corresponding to a possible solution, as the initial populations A, constituting the initial population space $I^{n}$.

Step 2: Evaluation and selection: Calculate the affinity function of antibody in the population, and divide $\mathrm{N}$ antibodies into two parts: candidate set $\boldsymbol{A r}$ and memory set $\boldsymbol{A m}$.

Step 3: Cloning: Clone k antibodies with the highest affinity, cloning number being proportional to the affinity.

Step 4: Mutation: According to a specified probability, randomly perform the operation of mutation on the cloned antibodies at a certain scale.

Step 5: Re-evaluation and re-selection: Re-calculate the affinity of antibodies cloned and mutated. If the affinity is even higher than the original, replace the original antibody with the new antibody, so as to form a new memory set.

Step 6: Extinction: Delete d antibodies with lowest affinity in Ar, generate randomly $\mathrm{d}$ antibodies, and add them to the population, in order to ensure the diversity of antibodies, which simulate $5 \% \mathrm{~B}$ cells naturally dying process in the biological clone selection.

Step 7: Stop: If the stop criterion is satisfied, then output the result, or else return to step 2.

Because high-order polynomial coefficient is appropriate or does not have a decisive influence on performance of linearization model of catalytic gas sensor characteristics, it directly affects the measurement result. Considering that clone selection algorithm has excellent optimizing performance, this paper uses the algorithm to tune polynomial coefficient with sample data of sensor calibration.

\section{USING ICSA TO OPTIMIZE POLYNOMIAL CO- EFFICIENT}

According to the characteristics of the sensor and improved clone selection algorithm, the block diagram using 


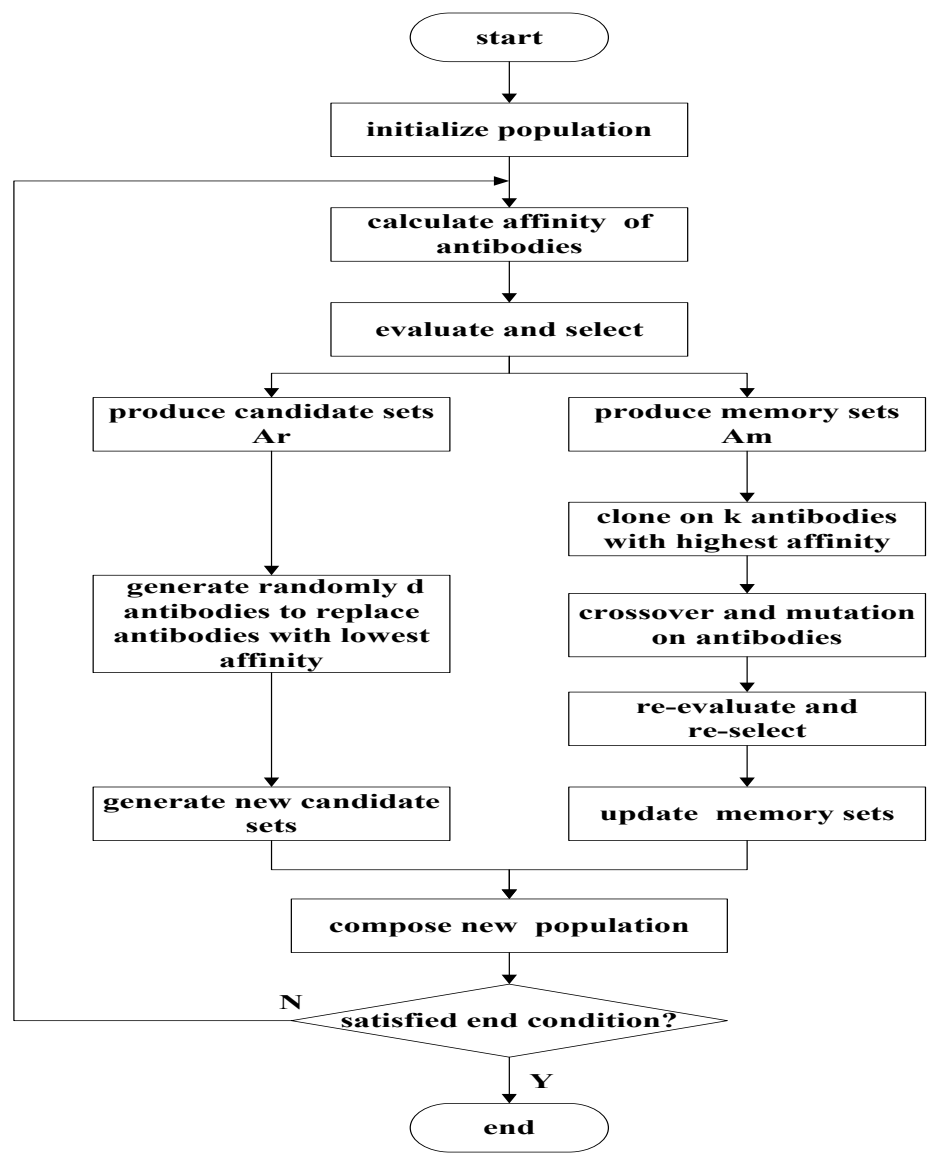

Fig. (1). Block diagram of improved clone selection algorithm.

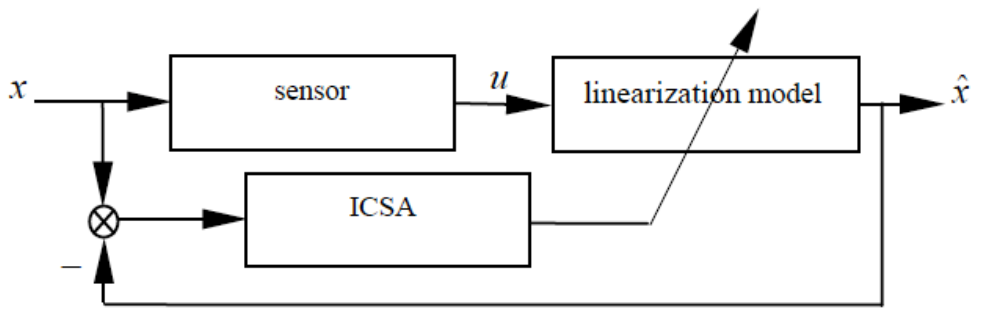

Fig. (2). Principle diagram of sensor characteristics linearization.

high order polynomial to fit characteristic curve is shown in Fig. (2).

In Fig (2), $\mathrm{x}$ is the input of sensor from the actual system, $\mathrm{u}$ is the sensor output, and $\hat{x}$ is the output of polynomial fitting model. The parameters of the fitting model are tuned intelligently by improved clone selection algorithm described above according to the criteria of mean absolute error minimization. The flow chart of tuning polynomial coefficients is shown in Fig. (3).

Being different from the conventional method using the traditional method of least squares, the modeling method in this paper does not employ the error sum of squares descent method to seek the optimal polynomial coefficients, but uses an improved clonal selection algorithm to search the optimal solution in the global scope, so as to get an optimal polynomial to establish inverse model of sensor.

\section{EXPERIMENT AND RESULTS}

To facilitate comparative research, this paper uses calibration data of catalytic sensor $0 \sim 4 \%$ of methane gas as the basic data resource [2], and adopts three order polynomial to fit output characteristics of sensor.

In order to make modeling error minimized and has good consistency, the mean absolute error is employed to evaluate the modeling performance. The expression of mean absolute error is shown in formula (2):

$$
\mathrm{MAE}=\frac{1}{n} \sum_{i=1}^{n}\left|\hat{x}_{i}-x_{i}\right|
$$

where, $\hat{x}_{i}$ is the output of model, $x_{i}$ is real value, $n$ is the number of sample. 


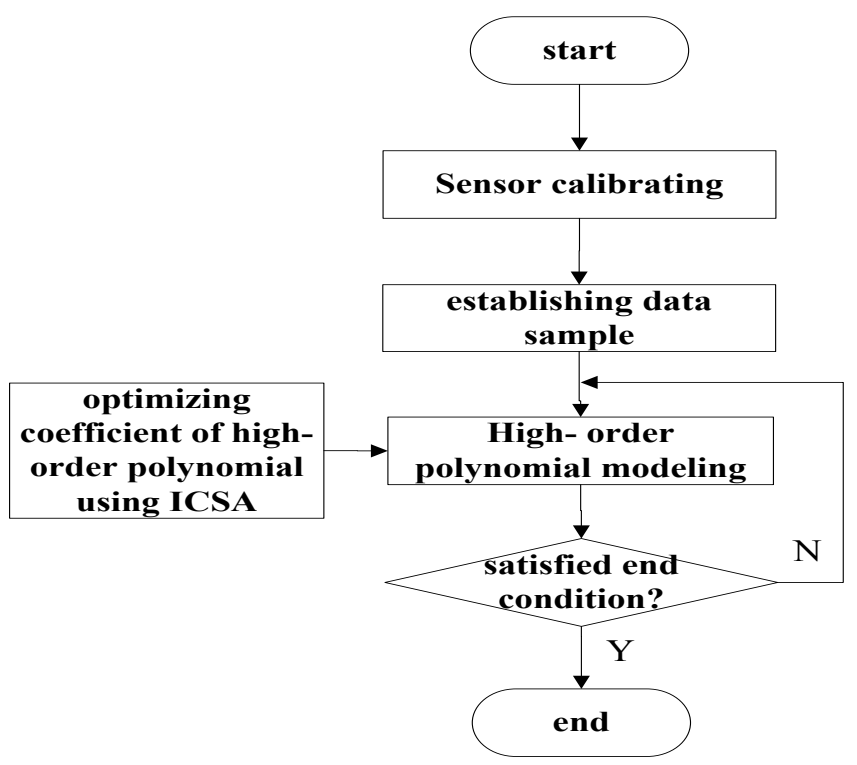

Fig. (3). Flow chart of tuning polynomial coefficients.

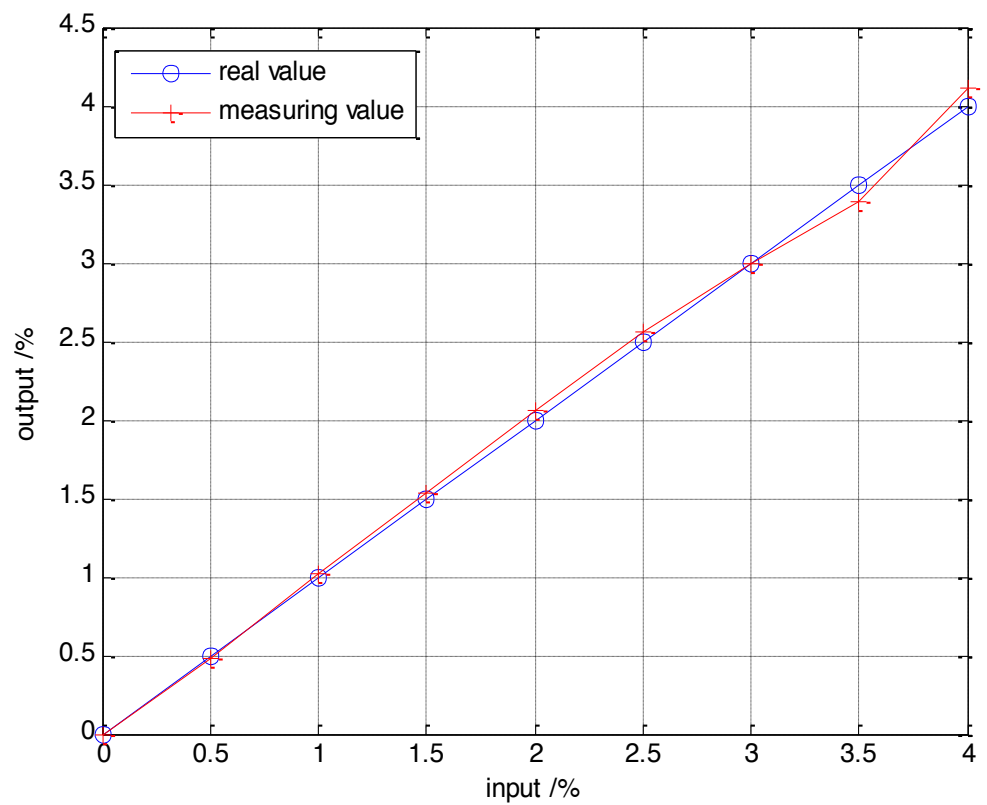

Fig. (4). Input-output curve of sensor.

To eliminate the effect of large range of the sensor output voltage on the modeling performance of the, the formula (3) is applied to normalize output voltage of sensor to the interval $[0,1]$.

$$
z_{i}=\frac{u_{i}}{\max \left\{u_{i}\right\}}
$$

where, $u_{i}$ is the sample value numbered $\mathrm{i}, \max \left\{u_{i}\right\}$ is the maximum value of $u_{i}, z_{i}$ is the normalized value of $u_{i}$, $i=1, \cdots, 9$.
Nine learning samples are used to train 3-order polynomial and the coefficients of polynomial are tuned by improved clone selection algorithm. The parameters of the improved clone selection algorithm are shown in Table $\mathbf{1 .}$

When MAE reaches to 0.04 , the algorithm stops running to get the optimal coefficient of the polynomial $\left(a_{3}, a_{2}, a_{1}\right.$ and $\left.\mathrm{a}_{0}\right)=(-0.009,-0.7487,4.8555,0.00000)$. The polynomial is expressed as follows:

$$
\hat{x}=-0.0009 u^{3}-0.7487 u^{2}+4.8555 u
$$

The polynomial above is the inverse model of sensor. After 9 training data are input into the inverse model, the final output results were obtained and listed in Table 2 . The inputoutput curve of sensor was displayed in Fig. (4). 
Table 1. Parameters settings of ICSA.

\begin{tabular}{|c|c|}
\hline ICSA Parameter & Setting \\
\hline \hline Total Generation & 100 \\
\hline Initial Population Size & 100 \\
\hline Length of Coding & 10 \\
\hline Clone Factor & 5 \\
\hline Suppression Threshold & 0.9 \\
\hline Cross Probability & 0.01 \\
\hline Mutation Probability & 5 \\
\hline
\end{tabular}

Table 2. Input-output data of sensor.

\begin{tabular}{|c|c|c|c|c|c|c|c|c|c|}
\hline Gas Real Value /\% & $\mathbf{0}$ & 0.5 & 1.0 & 1.5 & 2.0 & 2.5 & 3.0 & 3.5 & 4.0 \\
\hline Sensor Output Voltage/mv & 0 & 14 & 30 & 46 & 63 & 80 & 95 & 110 & 138 \\
\hline Gas Measuring Value/\% & 0.0000 & 0.4849 & 1.0201 & 1.5353 & 2.0605 & 2.5630 & 2.9874 & 3.3941 & 4.1059 \\
\hline
\end{tabular}

It can be seen from Table 2 and Fig. (4), the measurement results are very close to the true value. To evaluate the linearity of sensor, the independent linearity $\mathrm{L}_{\text {out }}$ is used as evaluation indicators.

$\mathrm{L}_{\text {out }}= \pm \frac{\Delta_{\mathrm{m}}}{\mathrm{X}_{\text {out-max }}-\mathrm{X}_{\text {out-min }}} \times 100 \%$

where, $\Delta_{\mathrm{m}}$ is the maximum absolute deviation; $\mathrm{X}_{\text {out-max }} \mathrm{X}_{\text {out-min }}$ is sensor range, which is the algebraic differential of the upper limit and lower limit of measurement.

From Table 1 and formula (4), $\mathrm{L}_{\text {out }}$ is $2.6475 \%$, while $\mathrm{L}_{\text {out }}$ is $4.2500 \%$ when using the least square method [2]. It can be seen that the linearization method proposed in this paper can improve effectively the linearity of sensor, and can get higher measurement accuracy.

On the other hand, the linearization method using least squares polynomial has $0.035 \%$ zero-error [2]. In order to avoid zero-error, the value of zero-error must be subtracted from the measurement result obtained, which not only increases error of the other measurement points, but also denies the theoretical basis of the method. While the linearization method proposed in this paper has no zero-error, does not have the defect of zero-point adjustment and can obtain higher measurement accuracy.

It must be noted that the method proposed in this paper does not increase the complexity of algorithm when the higher than 3-order polynomial is employed to establish nonlinear regression inverse model of sensor. And this method is also suitable for all kinds of one-dimensional or high-dimensional sensors.

\section{CONCLUSION}

In this paper, a new linearization method of carrier catalytic gas sensor characteristics using high-order polynomial was introduced. The optimal coefficients of polynomial were tuned by improved clone selection algorithm and the criteria of mean absolute error minimization. The experimental results show the effectiveness of the method. Compared to the traditional least square method, the method proposed in this paper can improve effectively the linearity of sensor, and can get higher measurement accuracy, especially being suitable for detection system of mine gas based on single-chip microcomputer.

\section{CONFLICT OF INTEREST}

The authors confirm that this article content has no conflict of interest.

\section{ACKNOWLEDGEMENTS}

This work was financially supported by the Open Foundation of Jiangsu Key Laboratory of Large Engineering Equipment Detection and Control (JSKLEDC201212), the Foundation of Basic Research Project (Natural Science Foundation) of Jiangsu Province of China (BK20131124), and Xuzhou Key Laboratory of Virtual Reality and Multidimensional Information Processing.

\section{REFERENCES}

[1] Z. Yu, Z. Y. Zhang, D. J. Xu, "Constant temperature catalytic gas sensor detection method and application in gas detection," Instrument Technique and Sensor, vol. 46, pp.126-128, 2007.

[2] K. H. Chen, W. K. Lu, "Research of function relationship between output voltage and gas volume fraction of carrier catalytic gas sensor," Industry and Mine Automation, vol. 39, pp. 92-95, 2013. 
[3] Z. Z. Zeng, W. Zhu, X. H. Sun, "Approach fitting the temperature characteristic curve of sensor with a high accuracy based on neural network algorithm,"Chinese Journal of Sensors and Actuators, vol. 20, pp.326-328, 2007.

[4] G. Liu, X. R. Liu, Y. H. Ji, "Nonlinear correction of methane sensor based on improved BP neural network," Transducer and Microsystem Technologies, vol. 27, pp. 15-20, 2007.

[5] W.Y. Huang, M. M. Tong, Z.H.R, "Using CPSO-SVM and data fusion to calibrate temperature characteristics of thermal sensor," Journal of Computer Applications, vol. 29, pp. 3259-3262, 2009.

[6] L. Sun, S. Y. Yang, "Fitting of non-linear relation of temperature sensor and reference temperature compensation based on LS-SVM, Journal of Applied Sciences, vol. 27, pp. 616-622, 2009.
[7] L.N. De Castro, F. J. V. Zuben, "Learning and optimization using the clonal selection principle," IEEE Transactions on Evolutionary Computation, vol. 6, pp. 239-251, 2012.

[8] X. J. Fang, L. S. Li, "Convergence proof for generic clonal selection algorithm", Application Research of Computers, vol. 27, pp.1683-1685, 2010.

[9] J.Y. Zhang, W. Zhang, Intelligent Fault Diagnosis and Forecast of Equipment, National Defense Industry Press: Beijing, pp. 116-121, 2009.

[10] L.D. Pan, D.Y. Li, J.Y. Ma, Principle and Application of Soft Measurement Technique, China Electric Power Press: Beijing, pp. $1-8,2009$.

[11] X.J. Bi, Information Intelligent Processing Technology, Publishing House of Electronics Industry: Beijing, pp. 310-330, 2010.

Received: September 16, 2014

Revised: December 23, 2014

Accepted: December 31, 2014

(C) Weiyong et al.; Licensee Bentham Open.

This is an open access article licensed under the terms of the Creative Commons Attribution Non-Commercial License (http://creativecommons.org/licenses/by$\mathrm{nc} / 4.0 /$ ) which permits unrestricted, non-commercial use, distribution and reproduction in any medium, provided the work is properly cited. 